\title{
El destino del enlace periodístico: percepción de los editores y aplicación práctica de la hipertextualidad en los medios españoles
}

\author{
Félix Arias-Robles ${ }^{1}$ \\ José Alberto García-Avilés²
}

Recibido: 2017-02-14

Enviado a pares: 2017-02-27
Aprobado por pares: 2017-04-04

Aceptado: 2017-04-25

DOI: 10.5294/pacla.2018.21.2.2

Para citar este artículo / to reference this article / para citar este artigo

Arias-Robles, F. y García-Avilés, JA. (2018). El destino del enlace periodístico: percepción de los editores y aplicación práctica de la hipertextualidad en los medios españoles.

Palabra Clave, 21(2), 275-309. DOI: 10.5294/pacla.2018.21.2.2

\section{Resumen}

La hipertextualidad sigue conformando la base de los contenidos digitales, pero la brecha entre la teoría y su aplicación práctica permanece abierta y casi inexplorada. Con el fin de arrojar algo de luz al anterior problema, esta investigación indaga sobre las divergencias entre tres ejes: 1) las aportaciones académicas sobre el uso de enlaces, 2) la perspectiva de los editores digitales y 3) su plasmación en los principales medios españoles. Para ello, tras una revisión teórica, se analiza el uso de enlaces en una muestra de ocho medios digitales con un análisis de contenido y mediante entrevistas semiestructuradas se profundiza en su desarrollo en las redacciones. Los resultados obtenidos revelan importantes contradicciones en la cantidad de enlaces que se utilizan y, sobre todo, la apertura de unos contenidos ex-

orcid.org/0000-0001-5910-1541. Universidad Miguel Hernández, España. farias@umh.es

2 orcid.org/0000-0001-5394-4804. Universidad Miguel Hernández, España. jose.garciaa@umh.es 
ternos que resultan escasos y poco específicos. Estos hallazgos confirman la necesidad de elaborar unas normas de estilo esenciales sobre la utilización de los enlaces que armonicen los hallazgos científicos con la perspectiva de los periodistas y, en especial, su práctica diaria.

\section{Palabras clave}

Hipertextualidad; periodismo digital; redacción periodística; producción periodística (Fuente: Tesauro de la Unesco). 


\section{The Fate of the Journalistic Linkage: The Perception of Editors and the Practical Application of Hypertextuality in Spanish Media}

\section{Abstract}

Hypertextuality is still the basis of digital content, but the gap between theory and its practical application is still wide open and practically unexplored. Aiming to shed some light on this, the present research investigates the divergences between three axes: 1 ) the academic contributions about the use of links; 2) the perspective of digital editors; and 3) their expression in the main Spanish media. To that end, and following a theoretical review, this paper analyzes the use of links in a sample of eight digital media with an analysis of content and through semi-structured interviews, delving further into their development inside the newsrooms. The results obtained reveal significant contradictions in the amount of links used and particularly in the opening of external contents that are scarce and not very specific. These findings confirm the need to elaborate essential style standards about the use of links that harmonize scientific findings with the perspective of journalists and, in particular, with their daily practice.

\section{Keywords}

Hypertextuality; digital journalism; journalistic writing; journalistic production (Source: Unesco Thesaurus). 


\section{0 destino do link jornalístico: percepção dos editores e aplicação prática da hipertextualidade na mídia espanhola}

\section{Resumo}

A hipertextualidade continua conformando a base dos conteúdos digitais, mas a brecha entre a teoria e sua aplicação prática permanece aberta e quase inexplorada. Com o fim de esclarecer isto, esta pesquisa indaga sobre as divergências entre três eixos: 1) as contribuições acadêmicas sobre o uso de links; 2) a perspectiva dos editores digitais; e 3) sua expressão nos principais meios de comunicação espanhóis. Para isso, após uma revisão teórica, analisa-se o uso de links em uma amostra de oito mídias digitais com uma análise de conteúdo e, mediante entrevistas semiestruturadas, se aprofunda em seu desenvolvimento nas redações. Os resultados obtidos revelam importantes contradições na quantidade de enlaces que se utilizam e, principalmente, a apertura de alguns conteúdos externos que são escassos e pouco específicos. Estas descobertas confirmam a necessidade de elaborar umas normas de estilo essenciais sobre a utilização dos enlaces que harmonizem as descobertas científicas com a perspectiva dos jornalistas e, especialmente, sua prática diária.

\section{Palavras-chave}

Hipertextualidade; jornalismo digital; redação jornalística; produção jornalística (Fonte: Tesauro da Unesco). 


\section{Introducción}

La forma de interactuar con cualquier interfaz informática, particularmente la web, pone de manifiesto la ubicuidad de la hipertextualidad. La navegación a través de nodos de información mediante botones o enlaces se encuentra en la raíz de gran parte de las plataformas digitales y de fenómenos más recientes, como la inmersión, la transmedialidad, la visualización de datos o la ludificación. El sino de los hipervínculos, más allá del aspecto que presenten, es perdurar e incluso florecer en el tiempo (Arias y García-Avilés, 2016a, pp. 49-50). Pero el destino al que conducen muchos de estos enlaces sigue presentando importantes interrogantes en el ámbito periodístico.

La hipertextualidad todavía ocupa una "posición privilegiada" (De Maeyer, 2011) en unos contenidos digitales cada vez más fragmentados (Cortés, 2010, p. 104). El enlace, desde hace años, se considera uno de los mejores métodos para acceder a la información (Kawamoto, 2003, p. 172; Díaz-Noci, 2006) y el eje integrador del lenguaje periodístico desarrollado en la web (Canavilhas, 2007, p. 6). Autores como Weinberger (2008, p. 187), Woo Park (2003, p. 49) o Jackson (1997) se muestran convencidos de que el enlace constituye el elemento esencia de la red. Domingo (2008, p. 118) considera que esto se podría trasladar a cualquier página web y a los textos periodísticos. En este ámbito, Beckett (2010) argumenta que la hipertextualidad añadía tres valores a las noticias: 1) diversidad editorial, 2) relevancia y 3 ) conectividad.

Sin embargo, la hipertextualidad no siempre se ha tratado con la profundidad y el rigor necesarios. En parte, por la incapacidad para recomponer una visión amplia sobre lo que se ha dicho y, al mismo tiempo, profunda sobre sus distintas implicaciones (McAdams y Berger, 2001). Diversos autores han criticado el abuso de un análisis de contenido cuantitativo basado solo en el recuento del número de enlaces. En muchos casos, a partir de modelos ideales (Rost, 2003, p. 170; 2006), tales investigaciones habrían intentado estudiar el desarrollo este fenómeno con métodos supuestamente empíricos que, en realidad, se basaban en especulaciones (Domingo, 2008, p. 121), a menudo con un exceso de determinismo tecnológico (Almirón y Jarque, 2008, p. 103). 
En esta línea, Deuze (2008, p. 199) lamentaba que gran parte de la investigación sobre el hipertexto periodístico se basara exclusivamente en los resultados obtenidos sobre las versiones digitales de los medios impresos. Larrondo (2007, 2010) y Larrondo y Díaz-Noci (2011) apostaron por la combinación de métodos cuantitativos con estudios cualitativos que incluyesen, por ejemplo, mapas conceptuales y también con la experimentación.

Pero la mayor parte de las propuestas se centraron en métodos como la entrevista. Steensen (2011,p. 11) y Dickinson, Matthews y Saltzis (2013) argumentaban que los estudios sobre periodismo digital se beneficiarían de una mayor contextualización y de un mayor foco en la práctica de los medios. Por eso, investigadores como Boczkowski (2005), Domingo (2008), Küng, Picard y Towse (2008), Steensen (2011), Weiss (2014) o Spyridou, Matsiola, Veglis, Kalliris y Dimoulas (2013, p. 76) sí han aplicado un enfoque más etnográfico.

No obstante, muchos de estos trabajos seguían sin resolver uno de los principales problemas de este campo de estudio: la divergencia entre la teoría y la práctica en la utilización de los enlaces, porque la adopción de la hipertextualidad, como otros procesos de innovación, está latente tanto en la teoría como en las contradicciones de las redacciones (Weiss y Domingo, 2010, p. 1168); y porque solo estudiando estos entornos, con una revisión previa de la academia y un análisis de los contenidos, se puede obtener una visión real sobre su uso. Por eso, esta investigación tiene como objetivo comparar la teoría académica, la perspectiva de los editores de los medios en línea españoles sobre las distintas formas de enlazar información y el estudio de los contenidos publicados en sus webs. En estos campos, los datos se agrupan en tres rasgos clave de los enlaces: 1) la cantidad de enlaces en el nodo de origen, 2) el género de los de destino y 3 ) la relación espacial entre ambos.

Con esta base, este trabajo se basa en la hipótesis general de que existe una importante brecha entre la perspectiva académica, la opinión de los editores y la aplicación práctica de los enlaces en los medios. Estas ideas se plasman en las siguientes hipótesis: 
H1: La academia y los profesionales consideran la hipertextualidad muy importante en el periodismo, pero los medios todavía emplean pocos enlaces en sus contenidos digitales.

H2: Los investigadores apuestan por usar más los enlaces externos, pero los editores todavía muestran reticencias, sobre todo cuando redirigen a la competencia, y esto se traduce en una preponderancia de los vínculos internos.

H3: Los trabajos académicos resaltan la importancia de enlazar a contenidos concretos, pero los profesionales no le dan importancia a este uso de los enlaces y, por tanto, no tienen en cuenta el género o el formato de los nodos a los que redirigen.

\section{Marco teórico}

\section{Uso de enlaces}

En los últimos años, se han realizado diversos estudios sobre la presencia de enlaces en los medios. Dimitrova, Connolly-Ahern, Williams, Kaid y Reid (2003) rastrearon las páginas de inicio de 26 cibermedios de 17 países para constatar que el hiperenlace se había convertido en un elemento extendido y consolidado. A partir de 72 cibermedios europeos, Van der Wurff y Lauf (2006) concluyeron que la hipertextualidad constituía el rasgo de la red menos desarrollada.

No obstante, la mayoría de estos análisis de contenido sobre enlaces constituyen instantáneas de la situación en momentos específicos y casi ninguno profundiza en aspectos concretos sobre su escritura. Los que sí tratan cuestiones como el número recomendable de enlaces de cada texto, por el contrario, raramente se basan en hallazgos cuantificables, pero sí en la intuición y la elucubración teórica.

La mayoría de estos trabajos señala que la clave se encuentra en lograr un cierto equilibrio entre la ausencia y el exceso: satisfacer cualquier demanda que genere la lectura, pero solo si añade valor al contenido (Salaverría, 
2005). Especialmente en contenidos complejos, habría que anticiparse a las necesidades del usuario mediante una labor de selección, edición y jerarquización (McAdams y Berger, 2001). Habría que tener presente que el enlace está aportando material adicional, no simplemente fragmentando un texto (Price y Price, 2002, pp. 145-148). Incluso se estimaba que un nodo que ocupe dos pantallas debería tener al menos tres enlaces (Landow, 2006, p. 200).

Algunos trabajos han apostado por métodos como la experimentación para analizar la mejor forma de aprovechar la hipertextualidad. La mayoría, como el de Madrid, Van Oostendorp y Puerta Melguizo (2009), comprobaba cuestiones como la influencia de los enlaces en la navegación o la comprensión, pero enfocados al diseño web o la documentación. En el ámbito periodístico, una investigación de este tipo, basada en pruebas con diferentes prototipos, llegó a la conclusión de que un mayor número de enlaces mejoraba los datos de navegación, la percepción del usuario y su comprensión de los contenidos (Arias y García-Avilés, 2016b, pp. 17-18).

Pero la principal carencia en este campo radica en la escasez de investigaciones que profundicen en su dinámica en las redacciones. La mayoría de los trabajos que lo abordaron se basaron en la realización de encuestas a profesionales. Los 138 periodistas digitales europeos interrogados por Quinn y Trench (2002) coincidieron en que los hipervínculos podían incrementar el valor de las historias, pero se mostraron escépticos sobre la capacidad del lector para calibrar la relevancia de estos nexos. O’Sullivan (2005) constató que concebían la hipertextualidad como una característica importante, pero que también se preocupaban por su capacidad para expulsar a los usuarios. Sin embargo, estos trabajos no iban más allá de sus opiniones generales e ignoraban, incluso, sus valoraciones sobre el trabajo diario en las redacciones.

Solo unos pocos investigadores han constatado las contradicciones entre la visión teórica de los profesionales y su puesta en práctica. De Maeyer (2012, pp.698-700; 2013, p. 744) descubrió que la hipertextualidad se asocia con valores como la transparencia, la credibilidad o la diversidad, pero 
que el entusiasmo sobre los beneficios contrastaba con el pragmatismo: los periodistas no lo usaban tanto como ellos mismos creían que era su obligación. Stray (2010) observó que, pese a reconocer el beneficio de la inserción de enlaces, los profesionales se mostraban "sorprendentemente lentos" al tomárselos en serio. No obstante, estos estudios también carecían de un análisis empírico que constatase estas divergencias en los contenidos publicados.

\section{Relación entre los contenidos de origen y destino}

La espacialidad de los enlaces y la relación entre los contenidos de origen y destino aglutinan la mayor parte de los estudios sobre la hipertextualidad en el periodismo. Tremayne (2006) detectó una disminución constante de la proporción de enlaces externos en el análisis que realizó sobre los diez principales medios estadounidenses. Quandt (2008) encontró, en un estudio de otros diez medios de los Estados Unidos y Europa, que 73 \% de los 1600 artículos analizados solo tenían vínculos internos. En esta línea, Himelboim (2010, p. 374) constató el escaso aprovechamiento que los medios seguían realizando de los enlaces externos. En Argentina, Negri (2010, p. 51) recogía la opinión del periodista Álvaro Liuzzi, que bautizaba como "síndrome del clic saliente" la resistencia a esta apertura de gran parte de los medios para retener el mayor tiempo posible al lector. Y recientemente en España, Fondevila y Segura (2012, pp. 32-33) detectaron que solo una de cada cinco portadas digitales contenía enlaces a otras webs.

Junto con estos análisis empíricos, se han elaborado trabajos teóricos que en general inciden en la importancia de los enlaces externos. Félix Bahón subraya que la hipertextualidad exter na constituye una "ventaja competitiva" para demostrar al lector que se le ofrece lo mejor del propio medio y del resto (Negri, 2010, p. 51). En esta línea, desde hace más de una década, se considera que no tener miedo a enlazar constituye una "señal de confianza" (Nielsen, 1999) y que estos hipervínculos proporcionan una mayor credibilidad (Wilde y Baschnagel, 2005) e imagen de marca (Noguera, 2012, p. 37).

Por el contrario, se argumenta que las reticencias a enlazar reflejan “egoísmo, aislamiento y cierta paranoia” (Price y Price, 2002, p. 152). Por 
tanto, los enlaces a fuentes consideradas como creíbles, especialmente a documentos o fuentes externas, mejorarían la calidad de la información digital (Carroll, 2010; Salaverría, 2005, pp. 69-70; González Pacanowski, 2008 , p. 534). Además, se considera que los enlaces influyen en la relevancia de los contenidos, incluso desde un punto de vista inmediato, por su influencia en los motores de búsqueda (Stray, 2010). Uno de los mayores defensores de la hipertextualidad externa es Jeff Jarvis (2007), quien critica el excesivo empeño de muchos medios por redactar versiones de informaciones ajenas en lugar de enlazarlas:

Cover what you do best. Link to the rest. That's not how newspapers work now. They try to cover everything because they used to have to be all things to all people in their markets. [...] But in the age of the link, this is clearly inefficient and unnecessary. You can link to the stories that someone else did and to the rest of the world. If you do that, it allows you to reallocate your dwindling resources to what matters [...]. In the rearchitecture of news, what needs to happen is that people are driven to the best coverage, not the 87th version of the same coverage. ${ }^{3}$

Sin embargo, existen importantes carencias en la conjunción de estas perspectivas con el estudio de la opinión de los profesionales y su actividad diaria. Navarro (2012, pp. 501-502) reunió las opiniones de un grupo de expertos quienes afirmaban que la confianza del usuario se incrementa si el medio enlaza fuera. Definían como "un acto de valentía y humildad" reconocer que otros pueden haber hecho un mejor trabajo; pero la perspectiva de los periodistas apenas se ha tenido en cuenta.

La investigación empírica sobre el género de los nodos de destino resulta prácticamente inexistente. Las principales referencias a estas variables aparecen en los trabajos más teóricos. La mayor parte de los autores coincide en que no todos los enlaces externos poseen el mismo valor. McAdams

3 Cubra lo que haga mejor. Enlace para el resto. No es así como funcionan los periódicos ahora. Tratan de cubrir todo, porque solían tener que tratar todos los temas para todas las personas de sus mercados. [...] Pero en la era del vínculo, esto es claramente ineficaz e innecesario. Se pueden enlazar a las historias que otro hizo y al resto del mundo. Hacer esto le permitirá reasignar sus reducidos recursos a lo que importa [...]. En la reestructuración de las noticias, lo que tiene que suceder es que las personas sean conducidas a la mejor cobertura, no a la 87a versión de la misma cobertura. 
y Berger (2001) consideran que estos hipervínculos siempre deben estar bien justificados. En esta línea, Rojas Orduña (2007, p. 108) se decanta por enlazar con las webs oficiales, porque están actualizadas y contienen datos fiables. Pero, además de pensar en la fuente, convendría tener en cuenta el contenido del nodo concreto al que se enlaza. Los enlaces localizadores (Cantalapiedra, 2003) o profundos, los que en teoría se recomiendan, son los que redirigen a páginas internas de otros sitios webs, no a sus páginas de inicio. Sí se han estudiado cuestiones como la creación de vocabularios documentales colaborativos en medios como elpais.com (Rubio, 2012), pero sin un enfoque sobre la hipertextualidad.

\section{Metodología}

La selección de la muestra para el análisis de contenido y las entrevistas se realizó a partir de diversos índices de relevancia, que se valoraron en función de su presencia en el conjunto de medios y los objetivos de esta investigación: el ranking Alexa, a través de su ranking de webs más visitadas y su medidor de enlaces recibidos, los datos de dos entidades dedicadas al estudio de audiencias en España, el Estudio General de Medios y la Oficina de Justificación de la Difusión y las cifras de comScore. Además, se contabilizaron los seguidores de los medios en Twitter y Facebook, las redes sociales más populares. Los medios finalmente seleccionados en la primera fase del muestreo estratégico fueron los siguientes: elpais.com y elmundo. es, versiones digitales de los dos medios impresos de referencia; lainformacion.com y elconfidencial.com, dos nativos digitales con modelos innovadores (De Lara, Arias, Carvajal y García-Avilés, 2015, p. 235); rtve.es y telecinco. es, las televisiones pública y privada con más tráfico en la red; y cadenaser. com y ondacero.es, las dos emisoras de radio con mayor audiencia en el momento del estudio.

Sobre esta selección, en primer lugar, se llevó a cabo un análisis de contenido (Igartua y Humanes, 2014, p. 14), para formular, a partir de ciertos datos, "inferencias reproducibles y válidas" (Krippendorff, 1990, p. 28) sobre los rasgos cuantificables (Wimmer y Dominick, 1996) del destino de los enlaces. Con un nivel de confianza de $95.5 \%$ y un margen de error de \pm 2 , se calculó una muestra mínima de 2398 textos. Un periodo de 190 días se 
consideró suficiente para obtener una visión amplia y sin sesgos estacionales. Para ello, se aplicaron métodos aleatorios, como realizar las mediciones un día cada dos semanas y alternar los días y las horas de observación (Krippendorff, 1990, p. 41).

En total, se extrajeron 14 muestras de cada uno de los ocho medios hasta alcanzar 112. El número de textos analizados ascendió finalmente a 2464. En cada observación, se seleccionaron 22 piezas de cada medio de diferentes plataformas: de la web, de blogs propios, de Twitter y de Facebook.

En esta muestra, el foco se centra en la unidad de estudio, el enlace, entendido como la conexión explícita entre uno y otro nodo o alguna de sus partes (Codina, 1997; Gunder, 2002, p. 112) y como el elemento que debe activar el usuario para que se materialice la hipertextualidad (Arias y García-Avilés, 2016a, p. 65). Para este trabajo, solo se tuvieron en cuenta aquellos hipervínculos situados dentro del texto que, de manera manual, el redactor incluyó en los márgenes de la pieza. Se descartaron, por tanto, enlaces como los que se emplean para la navegación dentro de cada medio. Finalmente, se identificaron y estudiaron 5234 enlaces a partir de tres variables: 1) la cantidad en cada pieza; 2) la relación espacial entre el nodo de origen y el de destino, es decir, los distintos tipos de enlaces internos y externos; y 3) el género del contenido al que redirigen. Todos los datos se estructuraron en una base de datos propia, se analizaron con el programa SPSS y se visualizaron a través de la aplicación digital Infogram.

A partir de esta misma selección de ocho medios, se estableció contacto con los responsables de contenidos de sus ediciones digitales, detallados en un anexo, y se concertó una entrevista con cada uno de ellos en sus redacciones. La entrevista semiestructurada permite acercarse al trabajo de los responsables de los medios en línea y obtener un mayor volumen de información, por lo que se considera esencial para entender la influencia de la tecnología en su actividad diaria (Paterson y Domingo, 2008, pp. 1-2; Mitchelstein y Boczkowski, 2009, p. 568). Los editores están vinculados de forma directa con una redacción con la que a menudo trabajan además 
de supervisar y coordinar. El cuestionario, disponible en un anexo (véase la página 309), estaba compuesto por ocho preguntas sobre los asuntos de interés para esta investigación.

\section{Resultados}

\section{Cantidad de enlaces}

Muchos de los hallazgos científicos coinciden con las opiniones de los periodistas entrevistados en esta investigación. Todos consideran el enlace como un elemento esencial en la reconfiguración de los contenidos periodísticos, tanto por su capacidad para ofrecer un mejor servicio al lector como por su utilidad para proporcionar notoriedad en los buscadores. Para estos profesionales, la posibilidad de enlazar es lo que distingue a un texto en internet y lo que mejora el servicio informativo. Para tres de los entrevistados, la hipertextualidad adquiere ahora mayor importancia, porque el usuario accede a los medios a través de piezas aisladas en las que debe resultar efectivo el diseño de navegación y la información ya no puede permanecer aislada.

La mitad de los editores destaca la importancia de la hipertextualidad en el posicionamiento en buscadores, el Search Engine Optimization (SEO). Son conscientes de que Google penaliza las "habitaciones vacías" y de que esta tendencia se acentuará en el futuro. Por eso, la mayoría señala que tratan de convencer a los redactores de que la hipertextualidad no es un capricho ni solo proporciona un servicio adicional al lector, sino que se trata de una manera de que las noticias se lean más y de que el usuario permanezca más en la página, lo que supone una mayor rentabilidad.

La mayor parte de los entrevistados destaca la asimilación, aunque con matices, de estas premisas en sus redacciones. En elpais.com, todos los periodistas trabajan para el papel y la web al mismo tiempo. Ellos se encargan de elaborar los enlaces de sus propios textos, aunque una mesa web realice después un control de calidad. Pero, en la práctica, conviven varias velocidades:

Hay gente muy digital que se vuelca y sus piezas están tan bien redactadas como editadas para la web y enlazadas; otros no tenían ni 
idea y han aprendido; a algunos les cuesta un poco más. Algunos vinculan demasiado y otros se quedan cortos (elpais.com).

En elmundo.es, los periodistas suelen estar sensibilizados con la importancia de la hipertextualidad, pero también existen diferencias entre la mesa de últimas noticias y los profesionales de secciones, más veteranos y especializados, pero menos familiarizados con las nuevas tecnologías. La mayor parte de los editores admite que carecen de unas normas de estilo establecidas, pero que el enlace se cuida desde la elaboración de la noticia hasta su edición. Solo elconfidencial.com llevó a cabo un proceso formativo con todos sus redactores para que no presentasen la información sin enlaces.

Algunos de estos editores admiten las dificultades que implica aprovechar convenientemente la hipertextualidad en el trabajo diario. Dos de ellos señalan que la carga cotidiana de trabajo les impide dedicarle el tiempo necesario, por lo que a veces se opta por publicar el texto plano para ir añadiendo enlaces poco a poco. Sobre todo, porque algunos redactores todavía no están realmente concienciados y consideran molesto este proceso. Para solucionar tales problemas y mejorar la calidad de los enlaces, se plantea la necesidad de formar a responsables de la edición hipertextual de los contenidos periodísticos.

Estas contradicciones entre la opinión general y la descripción de las rutinas profesionales se trasladan a los datos obtenidos en los medios estudiados. La mayor parte de los contenidos periodísticos emplea algún grado de hipertextualidad, pero el uso intensivo de este elemento todavía es poco común.

Un 28.49\% del total de los textos analizados carece de enlaces. Un $71.51 \%$, por tanto, contiene uno o más. La presencia de un único enlace (33.60\%) constituye el rasgo más común en la muestra estudiada. No obstante, las piezas que contienen dos $(12.78 \%)$, tres $(8 \%)$, cuatro $(4.18 \%)$ y cinco (3.41\%) también resultan habituales. Que 32.51\% de los artículos contenga entre dos y siete enlaces constituye un dato innegable sobre el desarrollo de la hipertextualidad, pero también es relevante que $82.87 \%$ 
de las piezas solo incluya tres o menos hipervínculos y que solo $9.54 \%$ de las piezas contenga más de seis (figura 1).

\section{Figura 1. Volumen de enlaces por pieza}

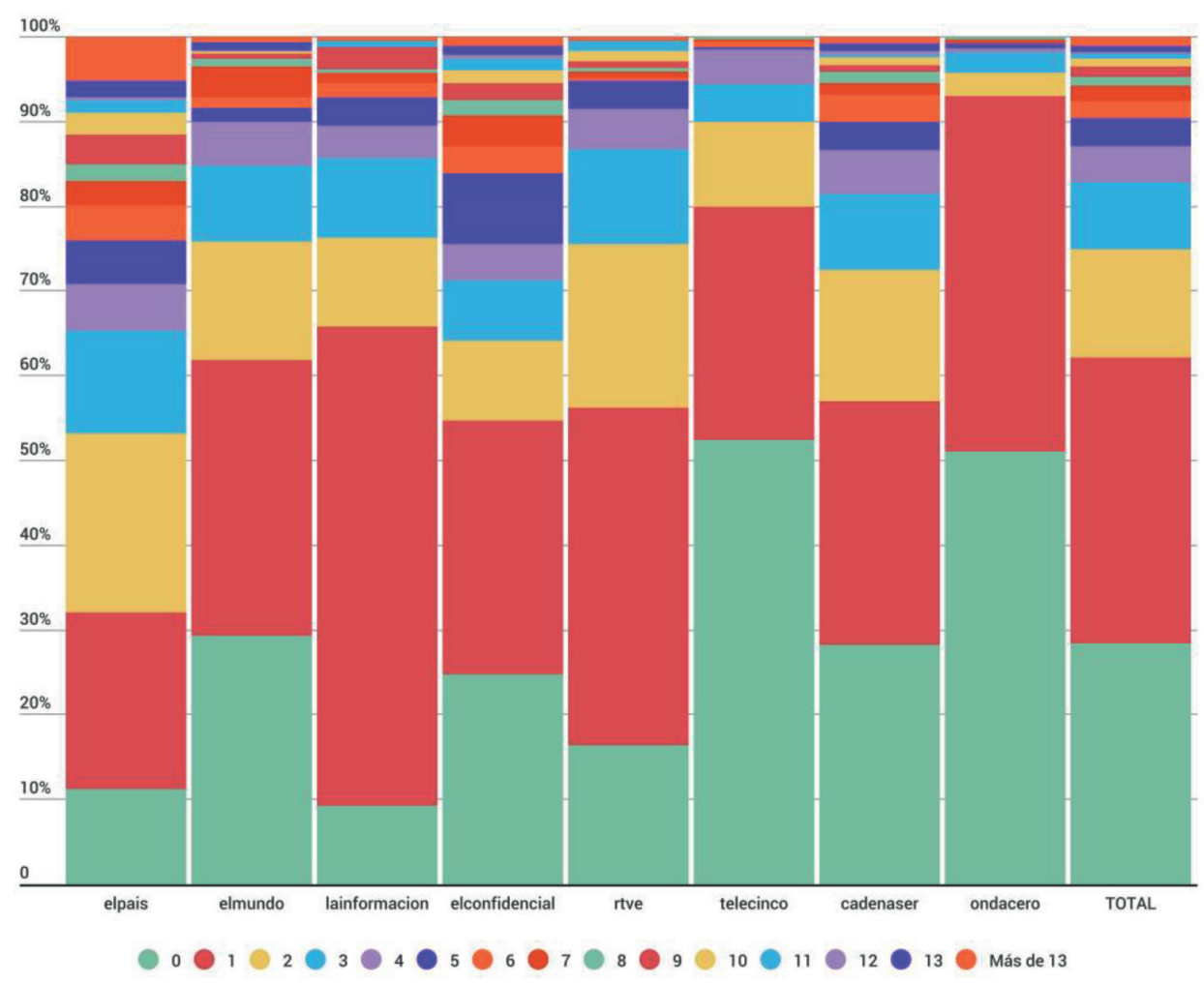

Fuente: Elaboración propia.

El estudio individualizado de los medios refleja que elpais.com constituye el medio con una hipertextualidad más desarrollada: solo $11.22 \%$ de las piezas carece de enlaces, mientras que $67.95 \%$ contiene dos o más y $5.13 \%$ más de trece. En segundo lugar, se encuentra lainformacion.com, donde hasta $24.74 \%$ de sus textos son planos, pero $24.47 \%$ tiene cinco o más hipervínculos. Por debajo, se sitúan elconfidencial.com, que registra el menor número de piezas sin hipervínculos $(9.28 \%)$ y rtve.es, donde 78.31 $\%$ de los textos tiene entre uno y cinco enlaces. En el lado negativo, destacan los casos de telecinco.es y ondacero.es, donde hasta $54.55 \%$ y $47.73 \%$ de sus piezas carece de enlaces. 
La figura 1 también revela diferencias entre los diversos tipos de matriz mediática. Los medios con raíz impresa y los nativos digitales presentan unas cifras más elevadas que las versiones digitales de las televisiones y, sobre todo, las radios. Además, muestra un patrón común: en cada tipo de matriz, existe un líder en el desarrollo hipertextual, especialmente en las televisiones y las radios.

La valoración de estos resultados por parte de los editores entrevistados varía entre los distintos medios, aunque la mayoría admite que tiene que mejorar. Los que obtienen datos más elevados, como elpais.com o lainformacion.com, consideran que se trata de cifras aceptables. Los que presentan unos resultados intermedios tienden a resaltar las acciones que están llevando a cabo para mejorar los procesos de inserción de enlaces. Finalmente, los que registran las cifras más negativas las atribuyen a limitaciones en los recursos humanos o los procesos técnicos disponibles.

\section{Nodo de destino}

La naturaleza del nodo en el que desemboca el enlace, como sucedía en las aportaciones académicas, constituye una de las cuestiones que mayor interés genera en los profesionales entrevistados en esta investigación. Todos ellos coinciden en resaltar la prevalencia del correcto funcionamiento de los hipervínculos internos, pero también son conscientes de la relevancia de los externos para el correcto desempeño de su labor informativa. Cuando estos enlaces desembocan en la competencia, no obstante, aumenta la controversia. Casi todos los entrevistados se muestran, en términos ideales, favorables a esta práctica. Sin embargo, algunos reconocen que, en el trabajo diario, por algún tipo de política empresarial o por falta de tiempo, suelen desatenderse más que el resto de hipervínculos.

Solo uno de los medios entrevistados, elmundo.es, admite que reescriben las exclusivas de otros y los nombran, pero sin ningún enlace externo. La costumbre y el temor a darle tráfico a la competencia constituyen sus explicaciones.

El resto adopta una postura más abierta, pero también más ambivalente. Los consideran positivos, pero admiten que las reticencias de algu- 
nos redactores y la falta de tiempo lastran esta práctica. Y cuando deciden abrir sus contenidos a la competencia, se muestran más exigentes que con el resto de enlaces.

Elpais.com no descarta el enlace a la competencia, pero califica como "absurdo" enlazar a otros medios por "algo que tiene todo el mundo" y añade que el mérito tiene que estar muy claro para concederle ese hipervínculo. Ondacero.es valora positivamente estos enlaces cuando sirven para atribuir o ampliar información, pero admite que casi nunca los introducen, porque la rapidez del día a día obliga a centrarse en los esenciales. Y en elconfidencial. com confiesan que los enlaces a otros medios constituyen "una pelea continua” en la redacción, aunque estén convencidos de la imparable generalización de este fenómeno:

La gente más veterana no enlaza a otros medios. De hecho, en la prensa española, se hace poquísimo si lo comparas con la anglosajona, donde no hay ningún tipo de prejuicio. Pero yo, como lector, quiero leer la información original. No tiene sentido no enlazarla. Es negarse a la evolución natural de las cosas. Al medio no le aportará ningún beneficio directo, pero el lector pensará que se lo está contando bien, que le está ayudando. Pero es verdad que entre el viejo periodismo hay un rollo muy duro de la competencia que cuesta romper. (elconfidencial.com)

Más claro parecen tenerlo en el resto de medios con matriz audiovisual. El miedo a perder al usuario existe, pero en cadenaser.com creen que el usuario volverá si recibe una información más completa. Desde su punto de vista, resulta inútil evitar estos vínculos, porque todo está a un solo clic en la red. Según cuentan, se trata de una costumbre que se ha normalizado y que "duele menos" en los despachos.

Hubo una época bastante oscura y viejuna con respecto a los enlaces externos. Parecía que el que oía La Ser no podía escuchar la Cope 0 RNE. En las ondas, esto podía ocurrir; en la web, es totalmente irreal. Siempre ha llamado más la atención entre los directivos que en la redacción. Pero nunca se nos ha pedido que quitemos un enlace. (cadenaser.com) 
Más allá de cuestiones de tráfico, en cadenaser.com creen que una de las grandes tendencias de la red es la prescripción, algo que siempre ha hecho la radio. Además, cuentan que en su radio recientemente han tenido buenas experiencias en el sentido contrario:

Cuando publicamos la última exclusiva, lo llevaron prácticamente todos los medios de comunicación. Y prácticamente todos nos enlazaban, como es normal. No esperas otra cosa. Es algo bastante habitual. Hace un par de años, sí que tenías que llamar a alguna redacción, a algún colega, para pedirle que te enlazasen. (cadenaser.com)

En esta línea, en telecinco.es, señalan que no hay que tener miedo, porque si el lector se va, será porque no se está haciendo bien en ese contenido concreto, porque no se da lo que se necesita. Y en rtve.es también opinan que el enlace a otros medios ya se ha interiorizado en las redacciones, aunque reconocen que quizá algo menos en empresas privadas en las que tienen que pelear directamente por audiencia y publicidad.

Los editores también consideran relevantes los hipervínculos que desembocan en el mismo nodo que el de origen, los denominados enlaces internodales o de ancla. En cadenaser.com, consideran que sirven para facilitar la lectura y duplicar las posibles puertas de entrada desde las redes sociales. Pero el uso de este sistema o la separación en dos de la noticia depende, sobre todo, de su contenido:

Hay temas en los que el despiece te pide estar dentro de la noticia, física y periodísticamente. Si es una noticia aparte, va a tener un titular con más entidad que un despiece. Un despiece con ancla es una aportación a la noticia principal, pero que no tiene todavía el carácter de noticia nueva. (cadenaser.com)

En rtve.es, aseguran que están intentando explotar estos hipervínculos en los últimos meses. Incluso para insertarlos en la portada, aunque desconocen si el usuario acaba de entender su funcionamiento.

A veces, generan una sensación rara, porque no encuentras el titular. En ocasiones, puedes no saber si se ha desplazado en la misma página o se le ha redirigido a otra distinta. Para evitar la confusión, solemos introducir una trama, pero aun así puede costar. (rtve.es) 
Una vez más, estas impresiones subjetivas apenas coinciden con la realidad. Los enlaces internodales adquieren un peso casi testimonial y los enlaces externos apenas alcanzan una cuarta parte del total (figura 2).

\section{Figura 2. Espacialidad de los enlaces}

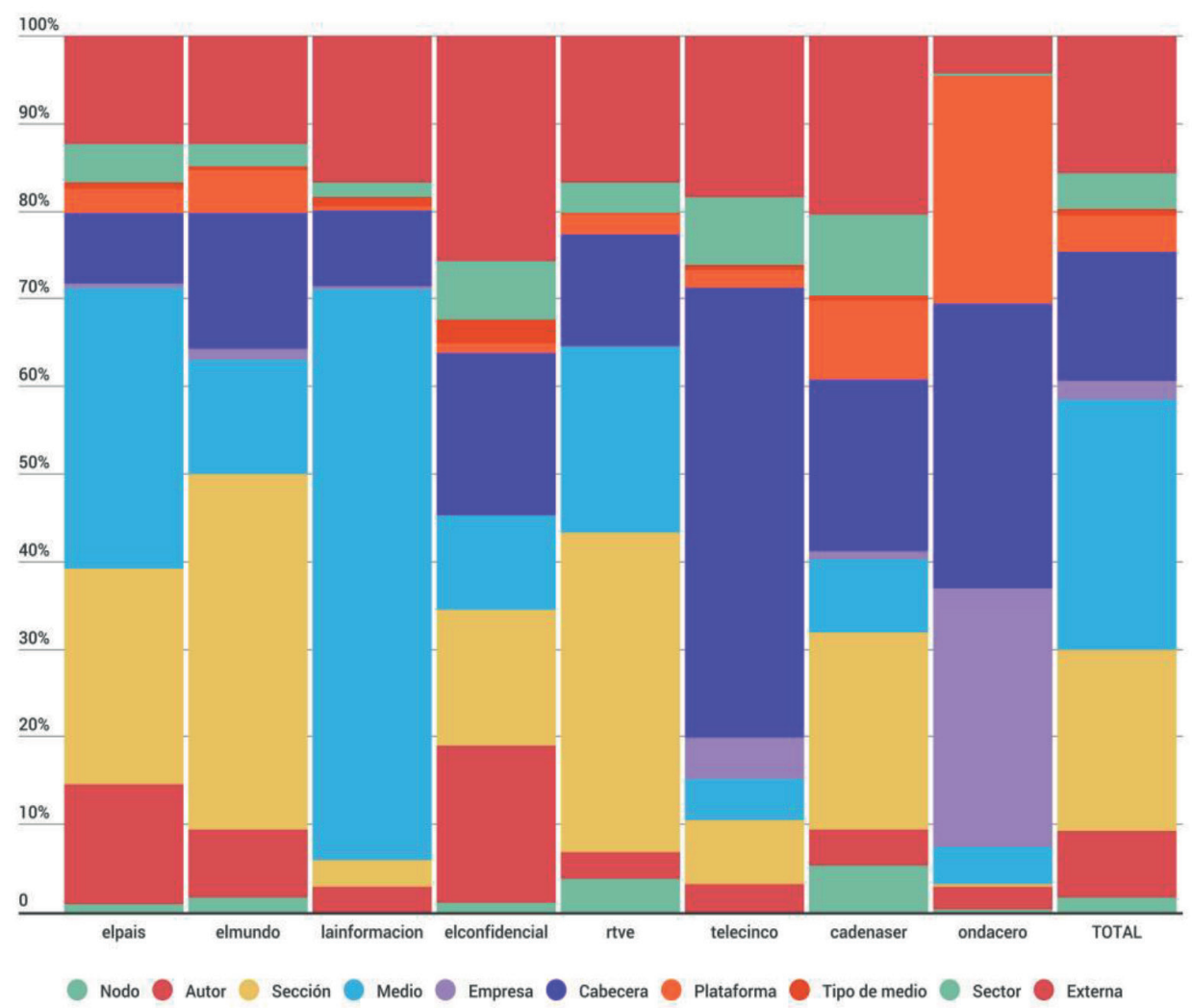

Fuente: Elaboración propia.

Los enlaces que redirigen al propio medio (28.62\%) son los más habituales, sobre todo por el uso de las etiquetas. A estos habría que sumarles los que no surgen de la misma sección (20.65 \%) y los que comparten un mismo redactor $(7.16 \%)$. Estos casos, generados siempre de forma manual, reflejan el carácter cerrado de un porcentaje importante de la hipertextualidad periodística.

También resultan relativamente comunes los hipervínculos que desembocan en la misma cabecera que emite el mensaje (14.69\%), los que se 
insertan en las redes para redirigir a los contenidos publicados en el medio o los que sirven para desplazarse entre la misma plataforma (4.07\%), principalmente las redes.

Los enlaces a plataformas externas al sector periodístico presentan unas cifras moderadas ( $15.71 \%)$. Sobre todo si se compara con la vinculación con informaciones de medios del mismo tipo de medio $(0.84 \%)$ y sector $(4.01 \%)$, los que verdaderamente constituyen referencias a la competencia.

Como en la tendencia general, los enlaces que desembocan en el mismo medio abundan en elpais.com (31.99 \%) y, sobre todo, lainformacion. com $(65.12 \%)$. Los nodos que comparten sección, en cambio, predominan en elmundoes ( $40.54 \%)$, rtve.es (36.49\%) y cadenaser.com (22.42\%). Entre los que enlazan a la misma cabecera, destacan telecinco.es (51.31\%) y ondacero.es (32.62\%), en especial por el peso que en estos casos poseen las redes sociales en la muestra.

Uno de los casos más reseñables es el de elconfidencial.com, donde los hipervínculos externos (25.68 \%) conforman el grupo más numeroso. No obstante, en el resto de medios, salvo en cadenaser.com (20.40\%), su porcentaje desciende hasta niveles tan bajos como los de ondacero.es (4.26\%).

Entre los que emplean enlaces internodales, solo puede resaltarse $c a-$ denaser.com (5.25\%) y rtve.es (3.79 \%). Los que comparten el mismo autor tienen una presencia mayor en elconfidencial.com $(17.89 \%$ ) y elpais.com (13.66 \%). Con mucha diferencia, ondacero.es es el que más enlaza a otros medios de su misma empresa (29.43\%), concretamente a Antena 3, aunque por cuestiones técnicas más que corporativas, como ellos mismos explican. Aunque representan un porcentaje mucho más reducido en cadenaser. com (1.01\%), en su redacción destacan la vinculación habitual con cincodias.com, al que enlazan desde la misma portada en las noticias especializadas en economía (figura 3).

La figura 3 agrupa, en los tres principales tipos de enlace, los datos anteriores. La preponderancia de los hipervínculos internos (73.75\%), que 


\section{Figura 3. Espacialidad de los enlaces (simplificada)}

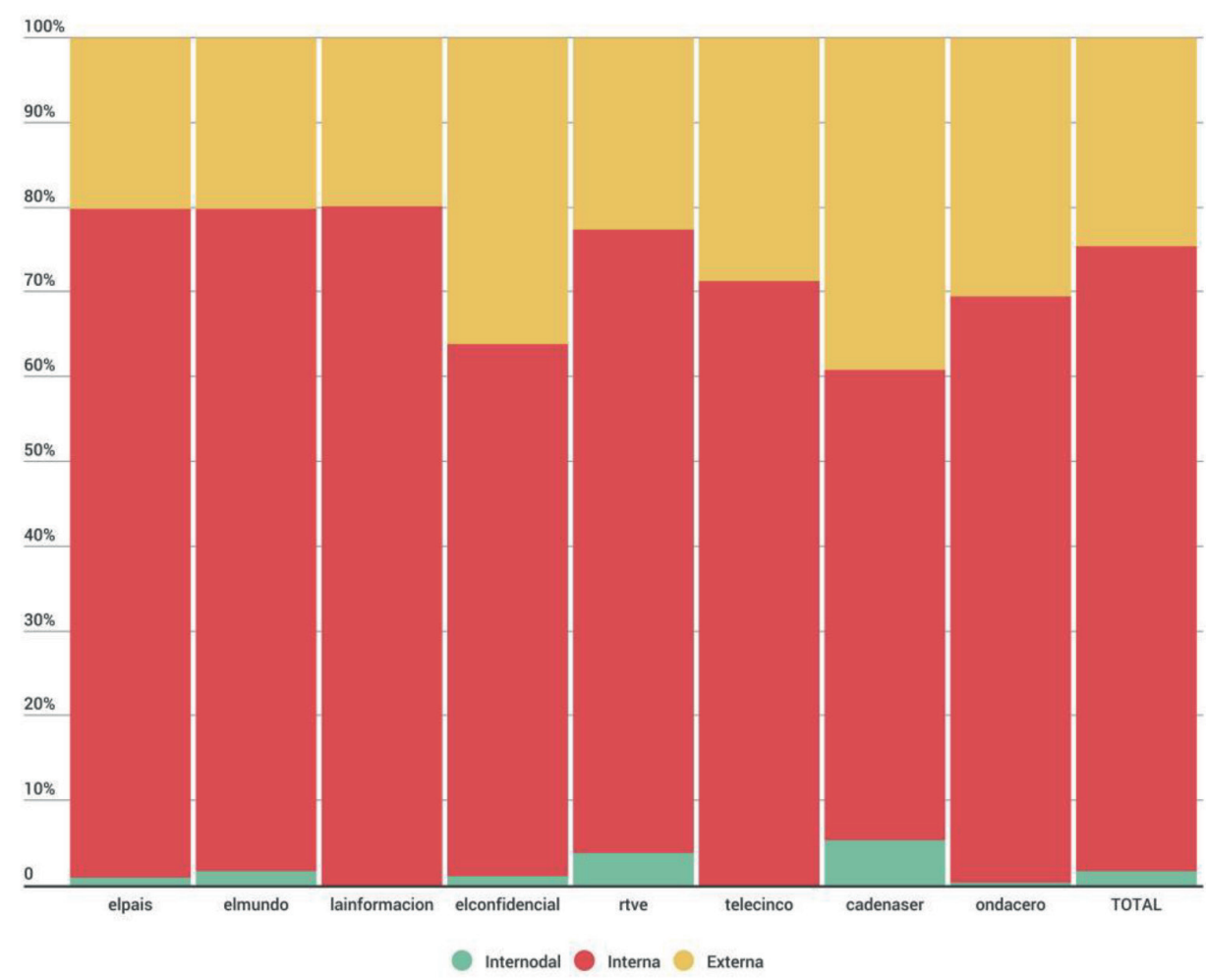

Fuente: Elaboración propia.

incluyen los del mismo autor, sección, medio, empresa y cabecera, resulta aquí más evidente. Los hipervínculos externos (24.63\%), que incluyen los de la misma plataforma, tipo de medio y sector, además de los externos, apenas representan una tercera parte de los internos.

Estos son los más comunes en todos los casos, pero existen diferencias sustanciales. Lainformacion.com (80.05\%), elpais.com (78.88\%) y elmundo. es $(78.04 \%)$ constituyen los medios más endogámicos. Los problemas de la prensa - y de algunos medios que en su día se presentaron como innovadores - para abrirse a la red, por tanto, siguen estando presentes. Por el contrario, elconfidencial.com (62.74\%) y, sobre todo, cadenaser.com $(55.56 \%)$ desarrollan una actividad hipertextual mucho más abierta. 


\section{Género de los nodos de destino}

El tipo de contenido al que redirigen los enlaces externos también despierta el interés de los editores. Todos destacan la necesidad de que proporcionen un servicio útil al lector con vínculos a destinos concretos y con valor informativo. En elpais.com, apuestan por enlazar con informaciones o documentos en lugar de páginas de inicio. En esta línea, elconfidencial.com considera esenciales los enlaces a documentos en PDF, porque dan una base de credibilidad y el público que cada vez demanda más fuentes primarias. Según señalan, a diferencia de lo que ocurría en la prensa, donde había que creerse lo que decía el redactor, ahora no citar un informe significa "casi engañar al lector o ser muy vago". Y esta opinión coincide con la de cadenaser.com, para quienes enlazar a fuentes primarias, como informes con datos, infunde confianza y permite al lector ampliar información:

\footnotetext{
Enlazar por enlazar, por ejemplo a un ministerio, tiene poco valor. Todo el mundo puede encontrarlo en el primer resultado de Google. Lo complicado es encontrar información pertinente, relevante y de valor. Ahí es donde los enlaces resultan vitales. (cadenaser.com)
}

Los entrevistados no se plantean si enlazan a un género periodístico concreto, pero sí tienen una opinión formada sobre uno de los principales tipos de enlaces: las etiquetas que desembocan en recopilaciones de artículos. Aquí las visiones de los entrevistados resultan más uniformes. Todos coinciden en la importancia de estos recursos, pero también se muestran autocríticos y convencidos de la necesidad de utilizarlos de manera conveniente.

Los responsables de contenidos de lainformacion.com y elpais.com, dos de los medios que más las utilizan, son conscientes de los excesos que a veces cometen en el uso de estos recursos. Sobre todo, cuando muchas etiquetas redirigen a temas poco relacionados con el asunto central de la noticia. Según explican, la creación de un sistema semiautomático de etiquetado pretendía mejorar su posicionamiento en buscadores, pero ahora dudan de su efectividad tras las modificaciones de sus algoritmos. Ambos señalan que debe bastar con tres o cuatro etiquetas adecuadas y que no se deben señalizar en el texto las que ya se incluyen en el margen superior. 
Otros, por el contrario, se lamentan por el escaso desarrollo de esta herramienta. Desde hace tiempo, en elmundo.es, cuentan con profesionales del SEO que marcan las palabras más buscadas en la red, pero ahora han creado un equipo para concretarlas y organizarlas. En cadenaser.com, opinan que este formato cada vez resulta más comprensible para el usuario. Más allá del SEO, se trataría de una manera lógica de organizar la información. Por eso, en lugar de limitarlo, aspiran a explotar con más contundencia este recurso.

Estas últimas opiniones contrastan con los datos obtenidos. Los enlaces a contenidos como informes o bases de datos resultan mucho menos frecuentes que los que desembocan en páginas de inicio. Los hipervínculos con nodos diferentes de los géneros periodísticos convencionales adquieren un peso considerable, lo cual podría reflejar la adaptación al nuevo escenario digital del lenguaje informativo. Sin embargo, esto se explica, sobre todo, por el peso que adquieren las etiquetas a recopilaciones de artículos, de las que parecen abusar diversos medios (figura 4).

Los géneros no periodísticos se han convertido en los contenidos más enlazados (43.06\%), como se detalla más adelante. Entre los géneros periodísticos, destacan las noticias $(28.01 \%)$, todavía esenciales en la narración de la actualidad. Del mismo modo, el peso relativo que también poseen los reportajes (7.32\%) o la opinión (6.06\%), por encima de los breves $(3.15 \%)$, demuestra el valor que, para enlazar, todavía poseen los contenidos más elaborados.

Entre los medios, se observan comportamientos muy diversos que se ajustan, en cierta medida, a la de sus distintos planteamientos periodísticos en la red. Los géneros no periodísticos resultan bastante frecuentes en todos los casos, pero su presencia alcanza cifras notables en ondacero.es (43.26\%) y, sobre todo, lainformacion.com (79.87\%). Entre los géneros periodísticos, también se detecta un predominio general de la noticia, con un peso mucho mayor en elconfidencial.com (45.68\%), elmundo.es (41.55\%) y telecinco.es $(38.74 \%)$. La entrevista resulta más frecuente en las radios ondacero.es (7.80 \%) y cadenaser.com (5.66\%); el reportaje, en los medios digitales con 


\section{Figura 4. Género periodístico de los nodos de destino}

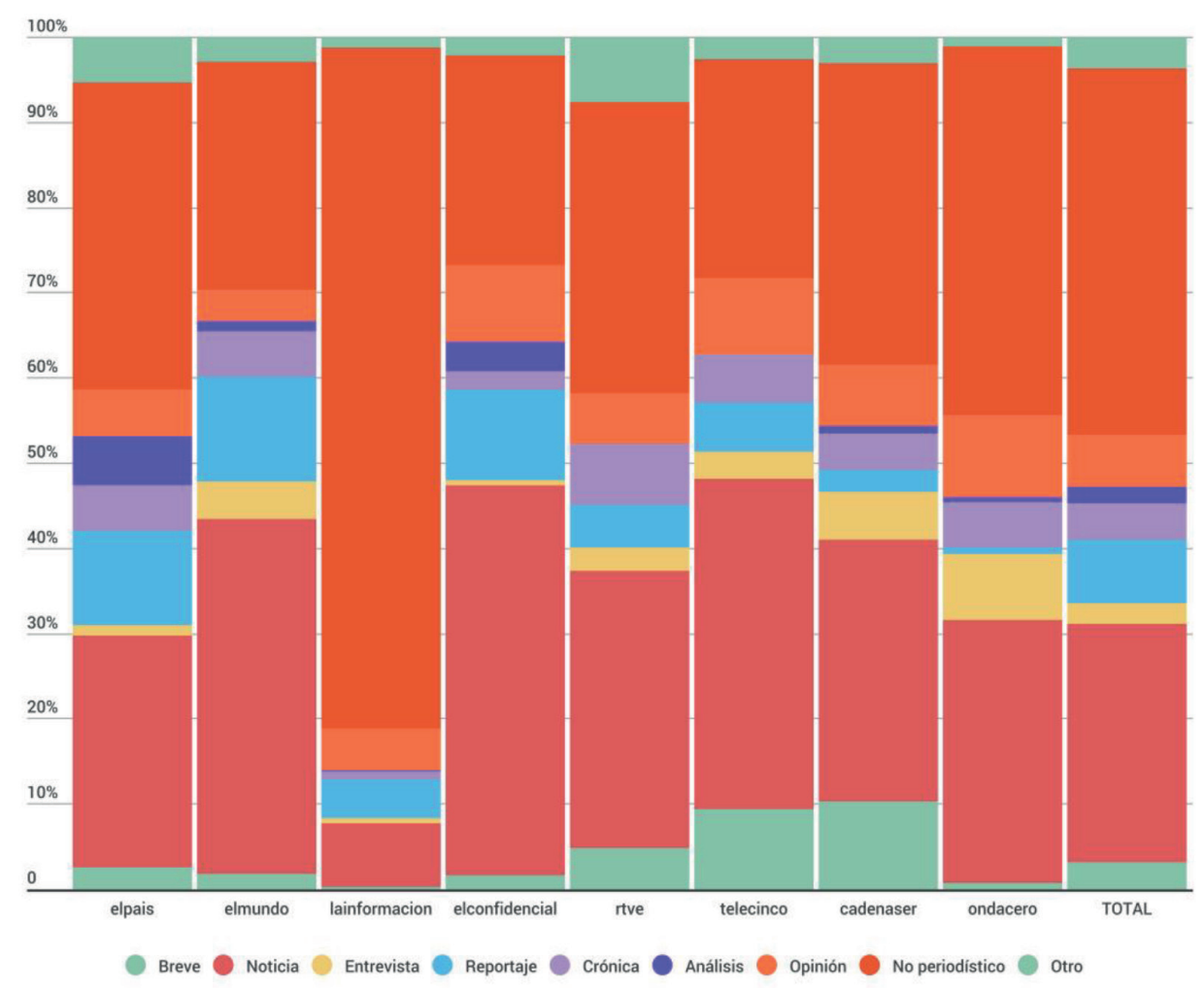

Fuente: Elaboración propia.

matriz impresa, elmundo.es (12,33\%) y elpais.com (11.18\%); la crónica, en rtve.es (7.06\%); el análisis, en elpais.com (5.82\%); y la opinión, en ondacero.es (9.57\%), elconfidencial.com (9.05\%) y telecinco.es ( $8.90 \%$ ) (figura 5).

El predominio de las recopilaciones de artículos (59.14\%) a las que en general se accede a través de las etiquetas resulta incuestionable. En un segundo escalón, se encuentran las páginas de inicio (15.48 \%), por lo común de entidades oficiales, así como los perfiles personales o profesionales de las redes sociales (13.53\%). Los enlaces a productos $(3.64 \%)$ o a bases de datos $(3.06 \%)$ también poseen un cierto peso relativo, pero el de otras categorías teóricamente importantes como los informes o documentos $(1.29 \%)$ resulta casi testimonial. 


\section{Figura 5. Género no periodístico de los nodos de destino}

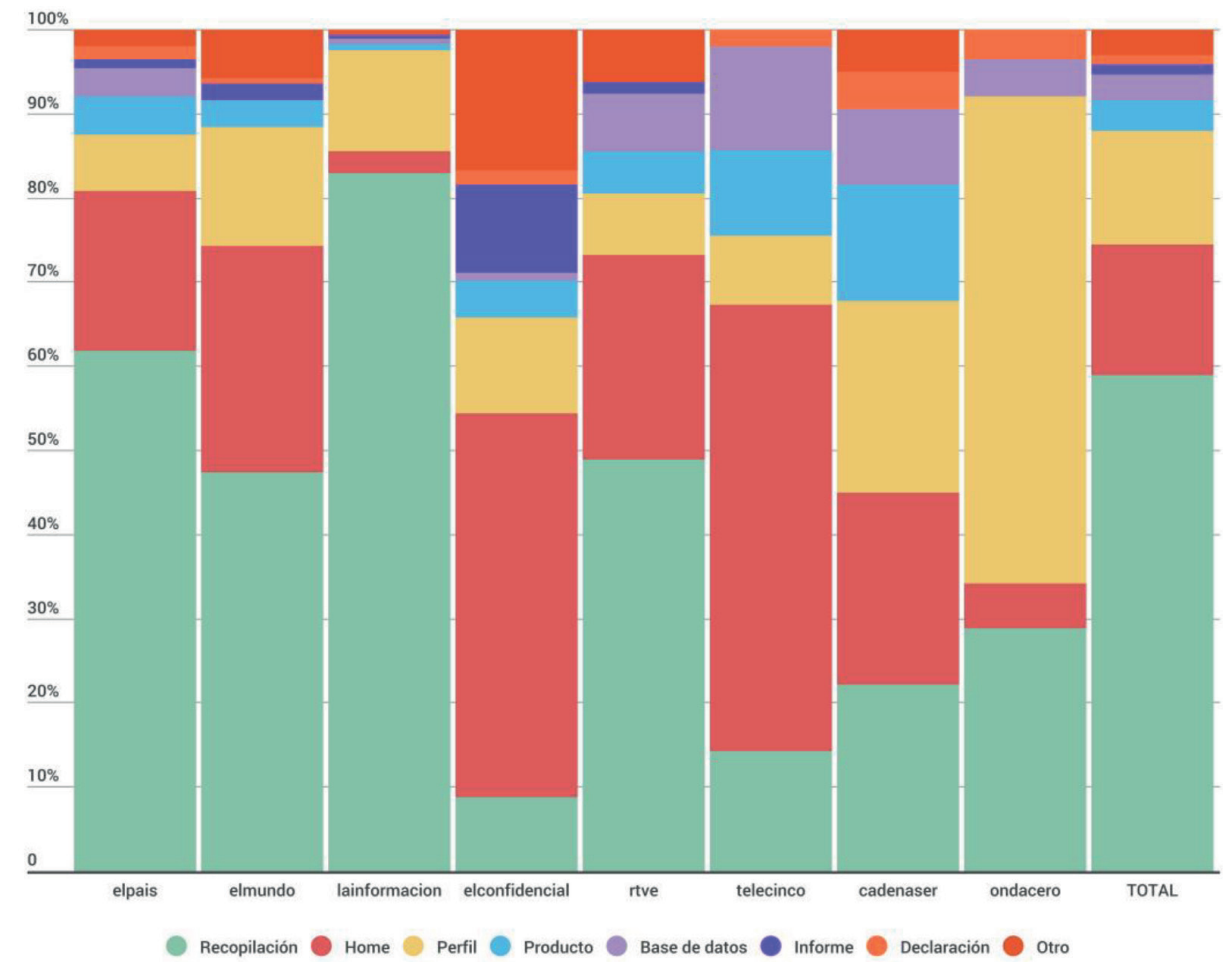

Fuente: Elaboración propia.

Las recopilaciones de textos a partir de etiquetas constituyen los principales destinos de la mitad de los medios estudiados: lainformacion. com ( $83.03 \%)$, elpais.com (61.85\%), elmundo.es (47.44\%) y rtve.es (48.97 $\%)$. En telecinco.es (53.06 \%) y elconfidencial.com (45.61\%), abundan más las páginas de inicio. En las radios, ondacero.es (57.89\%) y cadenaser.com $(22.78 \%)$ adquieren mayor relevancia los perfiles de las redes sociales. Los enlaces a bases de datos resultan más comunes en telecinco.es $(12.24 \%)$ y los informes en elconfidencial.com (10.53\%), lo que podría revelar un mayor desarrollo hipertextual.

\section{Conclusiones}

Los resultados obtenidos revelan importantes contradicciones entre los postulados teóricos sobre la hipertextualidad, la opinión de los editores 
digitales y el uso de los enlaces en los medios. Pero estas divergencias adquieren diferentes matices en función de los aspectos estudiados, así que conviene contrastar las hipótesis planteadas al inicio.

H1 se confirma. La hipertextualidad constituye, para los académicos estudiados y todos los entrevistados, un elemento clave en la reconfiguración de los contenidos periodísticos por su capacidad para mejorar la calidad de los contenidos y su visibilidad en los motores de búsqueda. Sin embargo, muchos de los profesionales admiten las dificultades que implica aprovechar convenientemente los enlaces en el trabajo diario y que no han llevado a cabo iniciativas para fomentar esta técnica en su redacción. Este hecho coincide con los datos obtenidos en diversos estudios generales sobre la presencia de enlaces en los medios, que, sin embargo, no profundizan en aspectos como la escritura ni su aplicación en las redacciones o carecen de base científica. Y esto se traslada a los resultados obtenidos en el análisis de contenido, donde se observa que la mayor parte de los contenidos periodísticos emplea algún enlace, pero raramente un uso intensivo de este recurso.

H2, en cambio, solo se confirma parcialmente. En efecto, los investigadores apuestan por un uso intensivo de los enlaces externos. Pero, en contra de lo que se suponía, esta visión también se ha extendido a la mayor parte de los editores de los medios estudiados. Salvo en unas pocas excepciones, reconocen que mejora la calidad del servicio y la credibilidad en un entorno en el que no tiene sentido establecer barreras. Esta opinión se matiza cuando los enlaces redirigen a la competencia: la mayoría apuesta por hacer referencia a ellos, pero solo cuando se trate de una información exclusiva. $\mathrm{Y}$ algunos admiten las reticencias de algunos directivos y redactores veteranos.

Esto explica, aunque solo en parte, que en la práctica los enlaces internos constituyen una clara mayoría. El hecho de que solo una cuarta parte de los hipervínculos sean externos confirma el largo camino de maduración que todavía le queda a la hipertextualidad periodística. No obstante, conviene destacar que estos resultados suponen una cierta mejora si se compa- 
ran con algunos de los análisis empíricos llevados a cabo en investigaciones anteriores. Además, hay que tener en cuenta casos concretos, como los de elconfidencial.com o cadenaser.com, en los que se observa una hipertextualidad mucho más abierta. Este contraste se traslada al empleo de los hipervínculos internodales, valorados positivamente en las redacciones, pero muy poco empleados en sus propios contenidos.

H3 también se cumple solo parcialmente; las contradicciones entre la teoría y la práctica se acentúan. La academia recomienda enlazar con contenidos relevantes y, en el caso de los externos, a las páginas internas. Esta perspectiva coincide con la de los editores, que síle dan importancia a este rasgo de la hipertextualidad y apuestan por enlazar con nodos como informes o documentos. Sin embargo, la realidad demuestra que las recopilaciones de artículos a partir de etiquetas o las páginas de inicio constituyen los géneros más habituales cuando los enlaces van más allá de los textos periodísticos convencionales.

Estos resultados confirman que, pese a la importancia otorgada a la hipertextualidad, todavía queda mucho camino por recorrer en la concordancia de la teoría con la práctica. Se necesitan trabajos que recopilen los hallazgos científicos, que recojan las opiniones de los profesionales y que analicen sus manifestaciones en los medios para mejorar el hipertexto periodístico. Y esta es la dirección en la que se mueve este artículo.

Sin duda, esta investigación presenta limitaciones que se podrían aprovechar para trabajos futuros. La muestra de medios analizados podría ampliarse, especialmente con la incorporación de medios más innovadores y de iniciativas de otros países. Y el análisis de contenido mejoraría con la inclusión de técnicas cualitativas como la realización de mapas conceptuales.

No obstante, los resultados obtenidos constatan la necesidad de crear unas normas de estilo esenciales a partir de la síntesis de los postulados teóricos y, sobre todo, de las necesidades y las prácticas habituales. A partir de investigaciones concienzudas y basadas en los datos, es necesario trasladar los principales hallazgos a los responsables de los medios digitales. Para eso, 
una de las vías más lógicas consiste en fomentar el intercambio de información entre la academia y la profesión en aspectos tan relevantes como la producción de contenidos. Evidentemente, estas claves deberán adaptarse a las particularidades de cada medio. Pero una vez alcanzado un consenso y obtenidos hallazgos firmes, convendría trasladar estas ideas a los redactores, con objeto de que perfeccionen el uso de un recurso tan esencial como los enlaces. Sobre todo, si se tiene en cuenta el lugar al que redirige el enlace. Porque ahí, en gran medida, se dirime su destino.

\section{Referencias}

Arias, F. (2016). Teoría y práctica del lenguaje ciberperiodístico: SEO, redes e (hiper)textos. Revista Mediterránea de Comunicación, 7(2), 177-194.

Arias, F. y García-Avilés, J. A. (2016a). Definiendo la hipertextualidad: análisis cuantitativo y cualitativo de la evolución del concepto. Icono14, 14(2), 48-68.

Arias, F. y García-Avilés, J. A. (2016b). Many short links. Digital Journalism, 1-21.

Beckett, C. (2010). Más cerca del ciudadano activo: el rescate del periodismo. Infoamérica: Iberoamerican Communication Review, 2, 45-57.

Boczkowski, P. J. (2005). Digitizing the news: Innovation in online newspapers. Cambridge: MIT Press.

Canavilhas, J. (2007). Webnoticia: propuesta de modelo periodístico para la $W W W$. Covilhã: Livros Labcom.

Cantalapiedra, M. J. (2003). Enlazar información. Hipertext.net, 1.

Carroll, B. (2010). Writing for digital media. Londres: Routledge.

Codina, L. (1997). H de Hypertext, o la teoría de los hipertextos revisitada. Cuadernos de Documentación Multimedia, 6. 
Cortés, L. V. (2010). Fragmentarismo y escritura: de la vanguardia a la metaliteratura. Sphera Pública, 10, 103-122.

De Lara, A., Arias, F., Carvajal, M. y García-Avilés, J. A. (2015). Ranking de innovación periodística 2014: selección y análisis de 25 iniciativas españolas. El Profesional de la información, 24(3), 235-245.

De Maeyer, J. (2011). Methods for mapping hyperlink networks. Comunicación presentada en el International Symposium of Online Journalism, Austin, Texas.

De Maeyer, J. (2012). The journalistic hyperlink: Prescriptive discourses about linking in online news. Journalism Practice, 6(5), 692-701.

Deuze, M. (2008). Toward a sociology of online news. En C. Paterson y D. Domingo (ed.), Making online news: The ethnography of new media production, 199-210. Nueva York: Peter Lang.

Díaz-Noci, J. (2006). Hipertexto y redacción periodística: ¿un nuevo campo de investigación? Razón y Palabra, 48(6).

Dickinson, R., Matthews, J. y Saltzis, K. (2013). Studying journalists in changing times. International Communication Gazette, 75(1), 3-18.

Dimitrova, D. V., Connolly-Ahern, C., Williams, A. P., Kaid, L. L. y Reid, A. (2003). Hyperlinking as gatekeeping: Online newspaper coverage of the execution of an American terrorist. Journalism Studies, 4(3), 401-414.

Domingo, D. (2008). When immediacy rules: Online journalism models in four Catalan online newsrooms. En C. A. Paterson y D. Domingo (eds.), Making online news: The ethnography of new media production (pp. 113-126). Nueva York: Peter Lang. 
Fondevila, J. y Segura, H. (2012). El peso de la hipertextualidad en el periodismo digital: estudio comparativo. Cuadernos de Información, 30, 31-40.

González, T. (2008). La revolución de los medios informativos en internet: el caso de los contenidos económicos. El Profesional de La Información, 17(5), 533-539.

Gunder, A. (2002). Aspects of linkology. Cybertext Yearbook, 1, 111-13.

Himelboim, I. (2010). The international network structure of news media: An analysis of hyperlinks usage in news Web sites. Journal of Broadcasting \& Electronic Media, 54(3), 373-390.

Igartua, J. y Humanes, M. (2004). Teoría e investigación en comunicación social. Madrid: Síntesis.

Jackson, M.H. (1997). Assessing the structure of communication on the World Wide Web. Journal of Computer-Mediated Communication, 3(1).

Jarque, J. M. y Almiron, N. (2008). Periodismo para internet o periodismo, y punto. Palabra Clave, 11(2), 219-252.

Jarvis, J. (2007). New rule: Cover what you do best. Link to the rest. En Buzz Machine.

Kawamoto, K. (2003). Digital journalism: Emerging media and the changing horizons of journalism. Lanham: Rowman \& Littlefield.

Krippendorff, K. (1990). Content analysis: An introduction to its methodo$\log y$. Berkeley: Sage.

Küng, L., Picard, R. G. y Towse, R. (2008). The internet and the mass media. Londres: Sage. 
Landow, G. (2006). Hypertext 3.0: Critical theory and new media in an era of globalization. Baltimore: JHU Press.

Larrondo, A. (2007). El reportaje hipermedia: análisis del género en los especiales de elmundo.es, elpais.com y lavanguardia.es (Tesis de doctorado, Universidad del País Vasco, Portugalete, España).

Larrondo, A. (2010). Propuesta metodológica para una aproximación empírica a los géneros ciberperiodísticos. Zer: Revista de estudios de comunicación, 15(29), 157-174.

Larrondo, A. y Díaz-Noci, J. (2011). La investigación del hipertexto periodístico: una propuesta de análisis. Ponencia presentada en I Congreso Nacional de Metodología de la Investigación en Comunicación, Asociación Española de Investigación de la Comunicación, Universidad Rey Juan Carlos.

Madrid, I., Van Oostendorp, H. y Puerta, M. C. (2009). The effects of the number of links and navigation support on cognitive load and learning with hypertext: The mediating role of reading order. Computers in Human Behavior, 25(1), 66-75.

McAdams, M. y Berger, S. (2001). Hypertext. Journal of Electronic Publishing, 6(3).

Mitchelstein, E. y Boczkowski, P. J. (2009). Between tradition and change: A review of recent research on online news production. Journalism, 10(5), 562-586.

Negri, I. (2010). El periodista digital como artesano de la información. En F. Irigaray, D. Ceballos y M. Manna (eds.), $2^{\circ}$ Foro de Periodismo Digital de Rosario (pp. 46-52). Buenos Aires: Fundación la Capital.

Navarro, F. (2012). Análisis prospectivo sobre la evolución de las herramientas de participación social en cibermedios españoles de diversa matriz 
mediática (Tesis de doctorado, Universidad Miguel Hernández de Elche, Alicante, España).

Nielsen, J. (1999). User interface directions for the web. Communications of the ACM, 42(1), 65-72.

Noguera, J. M. (2012). La innovación en el periodismo desde los medios sociales. En A. De Lara González, J. A. García Avilés, S. Martínez Mahugo y F. Navarro Maillo, Periodismo mutante: VII Jornadas Internacionales de Periodismo UMH. Murcia: Diego Marín.

O’Sullivan, J. (2005). Delivering ireland: Journalism's search for a role online. Gazette, 67(1), 45-68.

Paterson, C. y Domingo, D. (2008). Making online news: The ethnography of new media production. Nueva York: Peter Lang.

Price, J. y Price, L. (2002). Hot text: Web writing that works. Old Tappan: New Riders.

Quandt, T. (2008). (No) News on the World Wide Web? A comparative content analysis of journalistic news sites in four European countries. Ponencia presentada en Thinking Journalism Across National Boundaries, Montreal.

Quinn, G.y Trench, B. (2002). Online news media and their audiences. Heerlen: European Center for Digital Communication.

Rost, A. (2003). Una propuesta metodológica para estudiar el hipertexto en el periódico digital. Anàlisi: quaderns de comunicació $i$ cultura, 30, 169-183.

Rost, A. (2006). La interactividad en el periódico digital (Tesis de doctorado, Universitat Autònoma de Barcelona, Barcelona, España). 
Rubio, M. (2012). Nuevas destrezas documentales para periodistas: el vocabulario colaborativo del diario El País. Trípodos, 31, 65-78.

Salaverría, R. (2005). Hipertexto periodístico: mito y realidad. Tripodos, $67(1), 517-524$.

Spyridou, L.-P., Matsiola, M., Veglis, A., Kalliris, G. y Dimoulas, C. (2013). Journalism in a state of flux. International Communication Gazette, 75(1), 76-98.

Steensen, S. (2011). Online journalism and the promises of new technology: A critical review and look ahead. Journalism Studies, 12(3), 311-327.

Stray, J. (2010). Making connections: How major news organizations talk about links. En Nieman Journalism Lab. Recuperado de http:// www.niemanlab.org/2010/06/making-connections-how-majornews-organizations-talk-about-links/

Tremayne, M. (2006). Applying network theory to the use of external links on news web sites. En L. Xigen (ed.), Internet newspapers: The making of a mainstream medium (pp. 49-64). Oxford: Routledge.

Van der Wurff, R. y Lauf, E. (2006). Print and online news-papers in Europe: A comparative analysis in 16 countries. Amsterdam: HetSpinhuis.

Weinberger, D. (2008). The morality of links. En J. Turow y L. Tsui (eds.), The hyperlinked society: Questioning connections in the digital age (The new media world) (pp. 181-190). Michigan: University of Michigan Press.

Weiss, A. S. (2014). The digital and social media journalist: A comparative analysis of journalists in Argentina, Brazil, Colombia, Mexico, and Peru. International Communication Gazette, 77(1), 74-101. 
Weiss, A. S. y Domingo, D. (2010). Innovation processes in online newsrooms as actor-networks and communities of practice. $\mathrm{New} \mathrm{Me-}$ dia \& Society, 12(7), 1156-1171.

Wilde, E. y Baschnagel, M. (2005). Fragment identifiers for plain text files. En Sixteenth ACM conference on Hypertext and hypermedia (pp. 211-213). Salzburg, Austria: ACM.

Wimmer, R. D. y Dominick J. R. (1996). Mass media research: An introduction. Boston: Wadsworth.

Woo Park, H. (2003). Hyperlink network analysis: A new method for the study of social structure on the web. Connections, 25(1), 49-61. 


\section{Anexo}

\section{Preguntas del cuestionario}

1. ¿Qué importancia le otorgan a la capacidad para enlazar información?

2. ¿Qué estrategias están llevando a cabo para mejorar su utilización?

3. ¿Han desarrollado algún tipo de manual de estilo sobre su uso?

4. ¿Qué enlaces considera más importantes (internos o externos)?

5. ¿Utiliza enlaces que enlacen al mismo contenido de origen (intranodales)?

6. ¿Considera adecuado enlazar a la competencia? ¿Solo cuando se hacen eco de una exclusiva?

7. ¿Qué tipo de plataformas le parece más adecuado enlazar? (medios, blogs, redes sociales, webs oficiales...)

8. ¿Qué enlaces considera más útiles: los que llevan a una página de inicio o a un contenido concreto? 